\title{
Information policy and management framework for environmental protection organization with ecosystem conception
}

\author{
${ }^{1}{ }^{*}$ H. W. Chen; ${ }^{2}$ R. F. Yu; ${ }^{3}$ S. L. Liaw; ${ }^{3}$ W. C. Huang \\ ${ }^{1}$ Department of Environmental Engineering and Management, Chao-Yang University of Technology, Taiwan \\ ${ }^{2}$ Department of Safety, Health and Environmental Engineering, National United University, Taiwan \\ ${ }^{3}$ Graduate Institute of Environmental Engineering, National Central University, Taiwan
}

Received 24 October 2009; $\quad$ revised 3 February 2010; accepted 26 February 2010; avaiable online 1 March 2010

\begin{abstract}
Rapid development of information technology has changed people's attitudes towards information usage. To tender to the public's expectation, information system must feature facilities to increase the efficiency of information usage using modern information technology. Facing this challenge, it is necessary to establish a sustainable information environment, including information policy, data quality regulations and information management framework to deal with the rapidly increasing environmental data and changing behavior related to data/information usage except upgrading the hardware and software devices. Taking the uniqueness and complexity of environmental data into account, this study proposes a systematic framework based on the principle of life cycle assessment to outline the elements and its associated guidance required for a sustainable information environment. Simultaneously, the concept of information ecology is also embedded into such a planning for the purpose of establishing a self-evolutional information environment. Finally, the environmental protection administration of Taiwan is used as a case study to explain the practice of proposed framework.
\end{abstract}

Keyword: Data life cycle; Data quality system; Information ecology; Information flow

\section{INTRODUCTION}

For environmental status report and program management, Taiwan Environmental Protection Administration of Taiwan (TWEPA) spends a large portion of annual budget on environmental data collection. For shortening access time to environmental data, TWEPA concentrates on the implementation of the state- of-the-art technology (Cranefield and Purvis, 2001; Purvis et al., 2003) on integrating various environmental databases for data retrieval. Because of the costs involved in collection, data are deemed intrinsically valuable. TWEPA expends much time and efforts on its production and storage but less than adequate effort is made to promote usage of data by the public. Conforming to the trend of environmental information management (Haklay, 2003; Reddick, 2005), how to share existed massive environmental data to the public and partners in different governmental organizations and how to promote the efficiency of information usage with quality assurance (Huh, 1990; Wang et al., 1995; Wang

\*Corresponding Author Email: hwchen@cyut.edu.tw

Tel.: +886 42332 3000; Fax: +886 423742365 and Strong, 1996) have been critical issues in environmental information management (Garvey, 1998).Nominally, the collection and distribution of environmental data are centralized and undertaken solely by TWEPA. A user is to use whatever data offered by the government. The rapid development of information technology has made many changes possible in data production, usage and exchange (Nalle et al., 2004). Internet facilitates communication among multiple parties. Pictures, snapshots, popular writings and stories can be passed around easily. These not-so-academic environmental data are comprehensive to the public and can become crucial in the future. Civilians will be the primary suppliers of these data. One consequence of easy access to produce and utilize information is the enhancement if environmental literacy of the citizen will gradually accentuate the demand in environmental information both in depth and diversity. Data demand will be even more confounded as the computers assume the burden of computational and displaying drudgery and permit users devote more efforts in information 
interpretation and use. Therefore, prudence should be taken to incorporate the changes in data generation and information use in planning an environmental information system (Seifert, 2004; Gil-Garcia and Pardo, 2005; Reddick, 2005). As the changes of behavior, instrument, sources, role in environmental data/information usage for various users, environmental information ecology composed of individual, enterprise and governmental organization is forming (Abkowitz et al., 1999). Facing such facts, the role of government is not only in data collection and storage but also in data quality control and the promotion of data flow in an efficient manner either in public and private sectors (Ward and Mitchell, 2004; Scholl, 2006; Sofia Pereira and Soares, 2007; Mutula and Wamukoya, 2009), thus information economy will be emerged (Karmarkar and Uday, 2007).

Besides, the need of environmental information, either structured or unstructured data, for various environmental management problems such as environmental impact assessment has being extensive (Chen, 2009). In the past decade, the public has provided a large number of environmental data, information and knowledge. Given the fact, the public has been playing an integral role in environmental data supply chains. With the help of the public, some environmental actions, including environmental education, green consumption and knowledge management (Prokopiadou et al., 2004; Leire and Thidell, 2005; Huang and Shih, 2008) can be made further progress. Witnessing the rapid expanding of an information network and the changing role of participators, Steep (1999) proposed a model of information ecology, in which, the information management encompasses the issues of (1) linkages among production, distribution, interpretation and application of data/information (Steep, 1999; Kuchka, 2001) (2) dynamics of evolution in size and relationship diversity. Davenport and Prusak (1997) suggested that the information of an enterprise can be classed as informational, institutional and external environments categories based on the concept of information ecology (Davenport and Prusak, 1997). He stresses the establishment of procedures and channels that link among environment, information suppliers and consumers in order to improve the facileness of information flow, as well as to stimulate the information need of the public. Unlike general commerce services, an environmental information system addresses the advanced applications such as environmental education, knowledge management and decision-making. The role of government in environmental information management will be a builder for an efficient information environment rather than a successful data producer. The unique characteristics of environmental data makes users have to be concerned with the data quality. Thus, the quality assurance (QA) and quality control (QC) activities should be carried out when producing environmental data. Successful implementation of the data quality management system reduces organizational vulnerabilities in decision making and increases organizational credibility by providing the ability to make reliable, timely, cost-effective, and defensible decisions (USEPA, 2000a,b). Some mechanisms for controlling the quality of environmental data/information should be designed and emphasized while constructing an information environment comparing to the traditional information ecology (Davenport and Prusak, 1997). In 2000, TWEPA launched a ten-year action plan to integrate all environmental databases of central and local agencies by constructing a standardized environmental database system. The goal is to effectively provide useful and meaningful environmental information for many kinds and levels of users, including decision-makers of public and private sectors, researchers, students and general publics. To pursue the goal of a sustainable information environment, this paper proposes a series of strategy plans based on the report of TWEPA in 2002 (TWEPA, 2002) and to sketch an integrated framework of environmental information management and policy (de Brisis, 1995) for Taiwan government in the future by following the concept of ecology (Graham, 1985; Szaro et al., 1998). Information framework, basic elements in information ecology, normal regulations, standardized procedures, and organizational functions are re-defined in this study to provide managers with a referable template while planning or reconstructing an information environment by following the concept of information ecology (Moreton et al., 1995).

\section{MATERIAL AND METHODS}

Conceptualizing information behavior with ecology system

To outline the characteristics and regulations of 


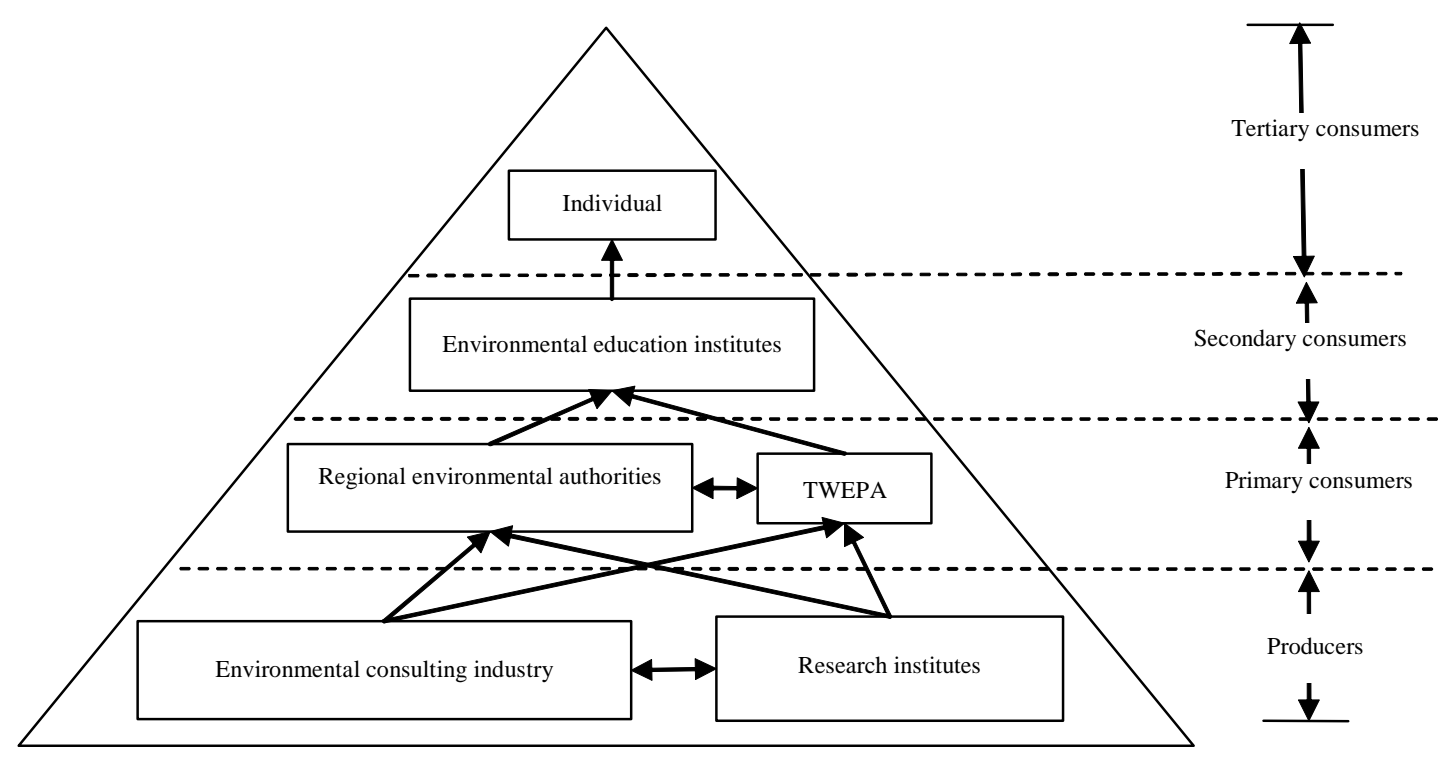

Fig. 1: Illustration of food chains in Environmental information ecosystem

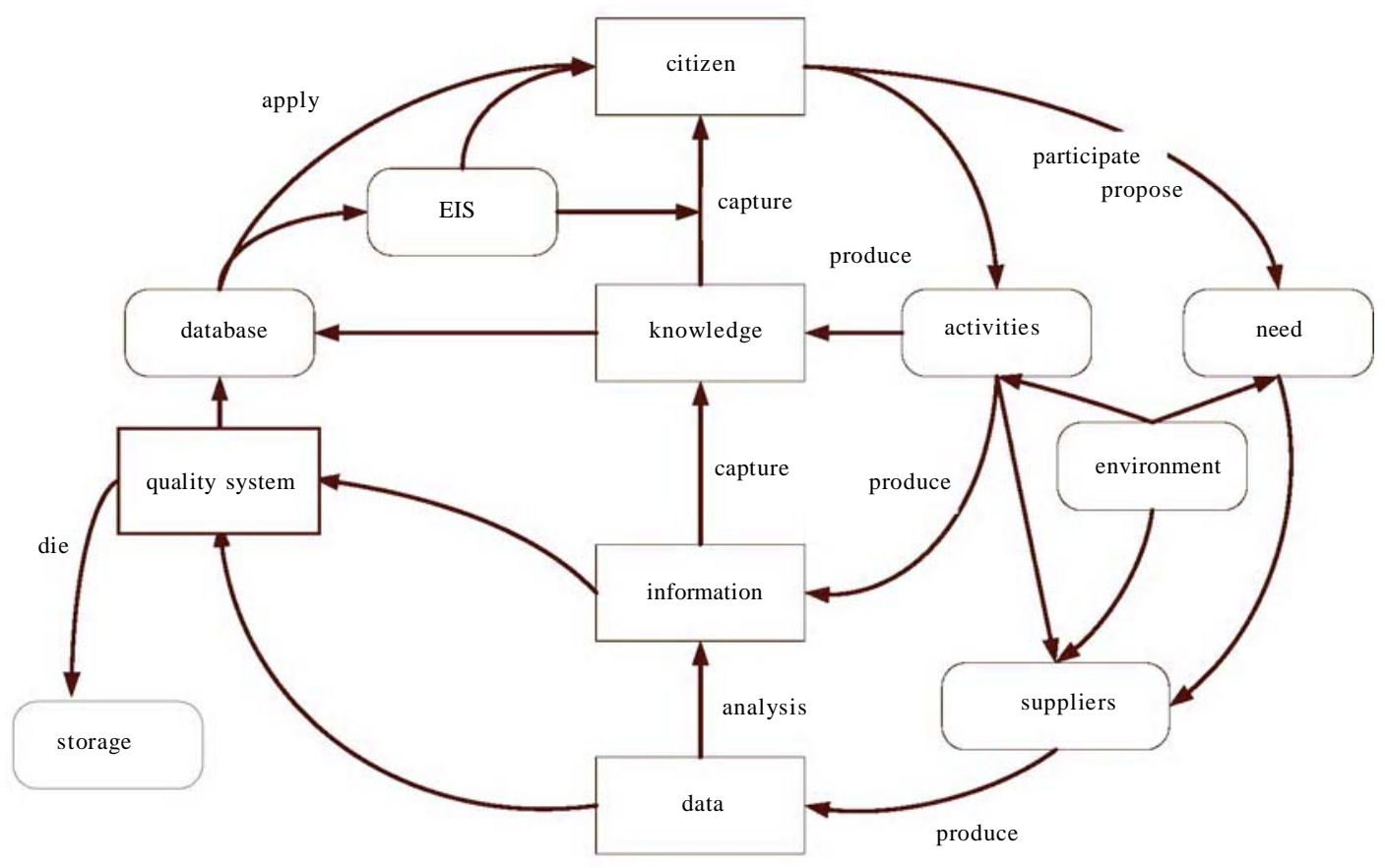

Fig. 2: Illustration of information chains in environmental information ecosystem

elements which comprise the environmental information ecology, Fig. 1 and Fig. 2 depict the organization illustrating information flow among suppliers, consumers and environment for an environmental information system, in which data disseminates, evolves and accumulates through positive and negative feedbacks between and among entities by emulating to the ecology system. These feedbacks are major booster for the evolution of information system. In Fig. 1, data/information is 


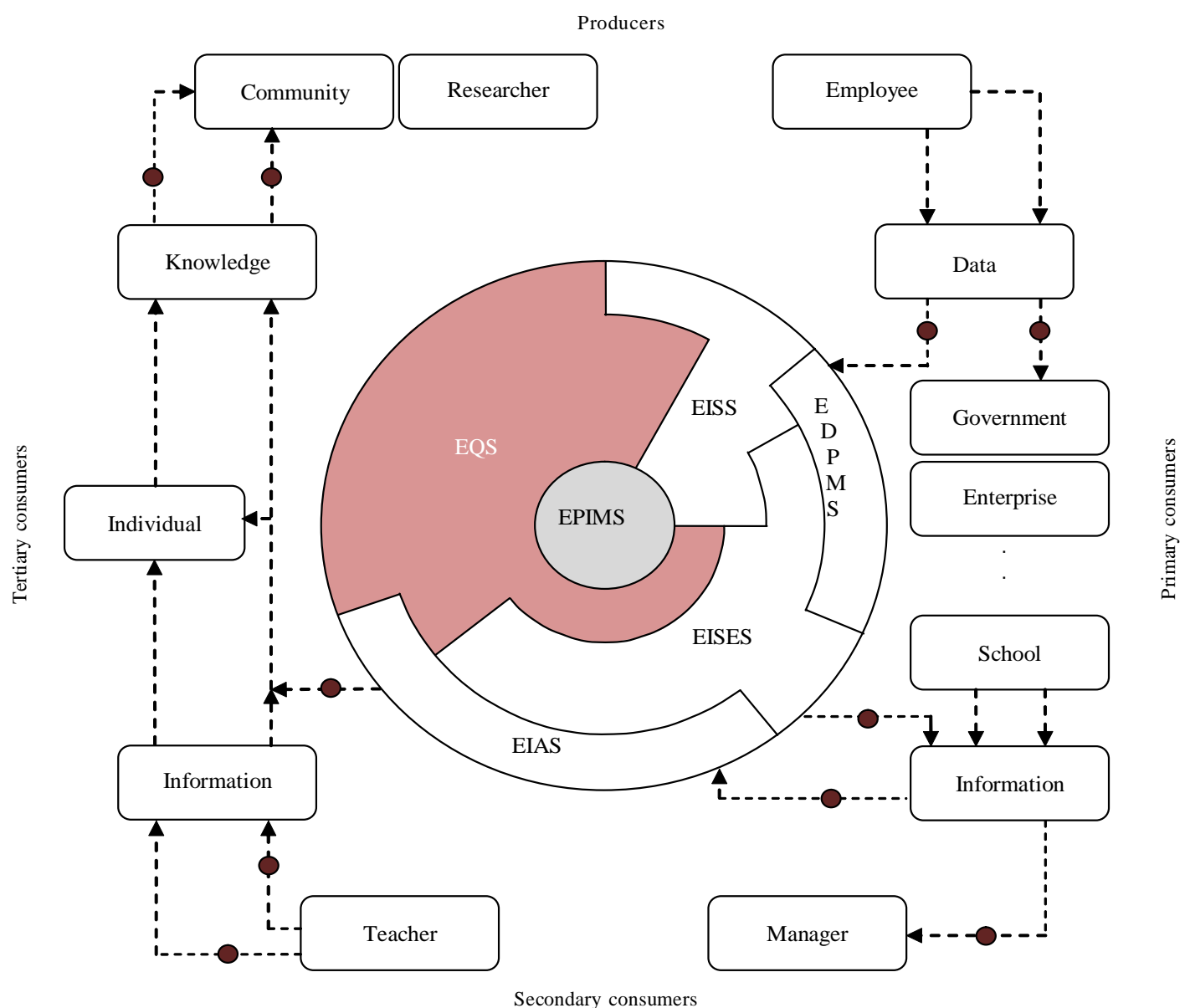

EPIMS: Environmental policy and institutional management system, EQS: Environmental quality system, EISS: Environmental information standard system, EISES: Environmental information supply and exchange system, EDPMS: Environmental data product management system, EIAS: Environmental information application system

Fig. 3: The framework of environmental information management system for TWEPA

considered as the basic nutrition and energy for the agents. Like energy in an ecosystem, environmental data is utilized and transformed into many forms, including theories, principles, rules and comprehensive knowledge as well as abstract concept and subjective meaning. The ability in capturing and generating data/information of a specific organism nearly preordain its life cycle and hierarchical level within food chains (Fig. 2). Within the environmental information ecology, the government is the main energy provider who shouldering the responsibility to draft reasonable regulations and to trigger off a series of evolution mechanisms, including competition, elimination, interaction and distribution for information evolution. Following such concepts, the function of government in information management should be tuned up to adapt to the incubation of information ecology. Accordingly, six components comprising environmental policy and institutional management system (EPIMS), environmental quality system, environmental information standard system (EISS), environmental information supply and exchange system (EISES), environmental data product management system (DPMS) and environmental information application system (EIAS) are suggested to TWEPA. These six components are expected to ensure the flow of environmental data stream in an efficient manner with quality assurance. As shown in Fig. 3, the components in the core, comprising, cooperate to evolve the 
Int. J. Environ. Sci. Tech., 7 (2), 313-326, Spring 2010

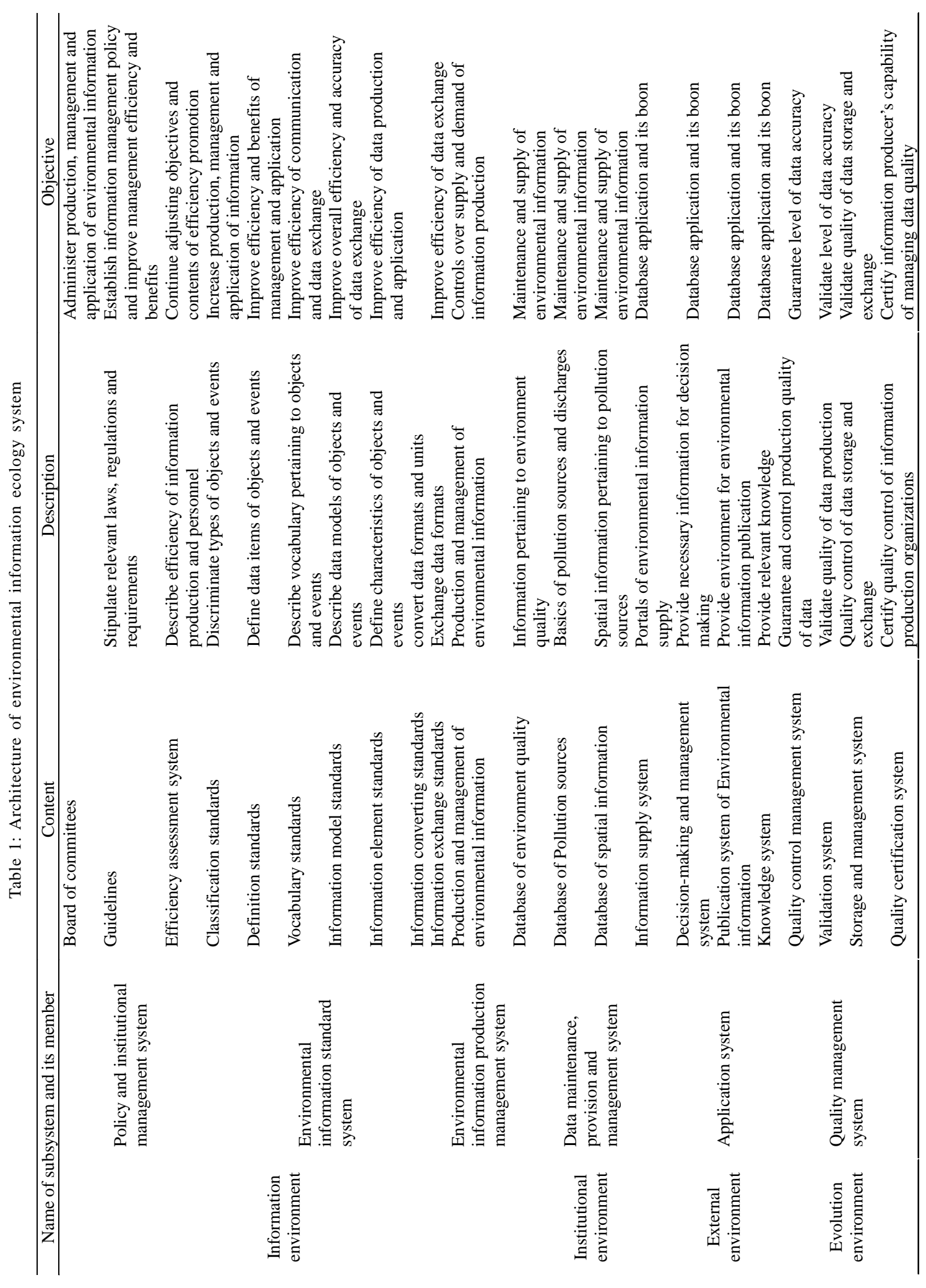


information environment and speed up the data/ information flow among agents. Applying the fundamental concept of ecology, the rational framework of environmental information system is as follows.

Framework planning of a sustainable information environment

Responding to the need adhere to the environmental information ecology, this research divides the information environment of TWEPA into four subsystems, including external, institutional, information and evolution system. Four subsystems and their respective members were included in Table 1 and discussed below.

\section{Planning of the information environment}

A well-functioning and open information environment must have the data scope, use policy, information standards and management procedures established. To draw up reasonably information policy and guidelines with consideration given to managing personnel, policy making and public education realm, EPIMS has been set up for TWEPA in 2001. As shown in Fig. 3, the EPIMS is not only the core of environmental information management system of TWEPA, but also the promoter of environmental information ecology. Additionally, an efficiency assessment system is also established for continue adjusting objectives and contents of efficiency promotion.

Fig. 4 shows the designed organizations and the associated responsibilities of data management for TWEPA. The Environmental Information Promotion Committee (EIPM) is comprised of three subcommittees, the environmental data standard subcommittee (EDSS), environmental data quality subcommittee (EDQS) and environmental data publication subcommittee (EDPS). They are assigned to control the quality and usage of data/information in the course of data production, storage, and dispersion, together with technical support to the TWEPA organizations. Since most environmental data collection and supervision is conducted by the departments/offices of the TWEPA and the local EPB, a specific quality management program (QMP) is required for each department, office and local EPB. Concept of natural selection foretells that the chances of producing offspring for the inept species or individuals are rare as competition occurred while environment becomes stringent. To facilitate the system evolution through competition and corporation, it is sound to establish a healthy hardware and software environment, which is accessible to information agents. Generally, the software components include the facilities for information acquisition, data handling and elaboration for knowledge accretion, and the hardware are to provide supports for software and data application. Thus, environmental information standard systems is built to facilitate data transportation and transformation, in which various standards such as data classification, environmental objects definition, environmental vocabulary, information model, environmental information element, information converting and information exchange are regulated. The descriptions and objectives of these standards are listed in Table 1 . The standard system is the very base of the whole distributed environmental database system. It includes the regulations, standards and guidelines required for establishing and operating the integrated database system. In addition, a standard and reference registry system of environmental terms and data elements should also be built for data exchange and integration. To ensure all of the data is conformed to the requirements of environmental quality and standards before spreading around to consumers, EDPMS is planned to regulate the actions about data production and storage. Fig. 5 shows the conceptual diagram of the proposed framework. The data collection system aggregate must be able to various types of environmental data and information generated by central and local governmental organizations. Accordingly, the information sources management system is designated as a registry system for registering the information.

The database system is the central part of the whole system, which should be an internet-based database system, but currently built with different purposes and database models mostly. For this reason, an adequate data model will be selected or developed in accordance with the data element and term standards for data integration. The information application system includes various information systems with certain purpose, such as environmental management information system for governmental decision makers, environmental information and 


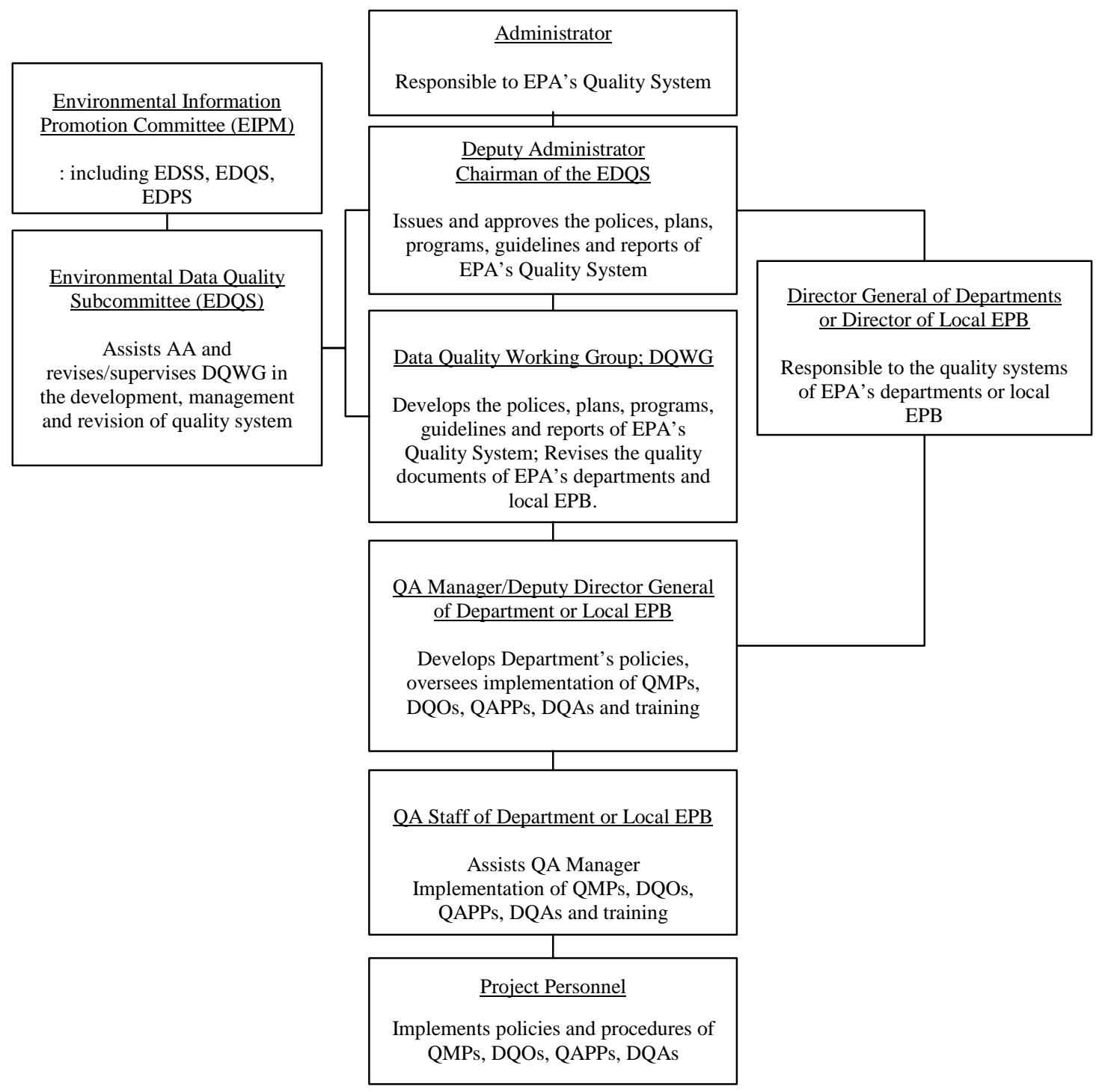

Fig. 4: Organization and responsibilities of data quality management for Taiwan EPA

knowledge system for general publics and students, environmental analysis system for system analysts and researchers and so on.

Planning of institutional and external environments Interaction and distribution mechanism promote smooth data flows and information diversity. Interaction mechanism can be established via cooperation between private sectors and academic institutions and integration of information production and applications. BBS and discussions among community members in a chat room and other instant communication platforms can be alternative tools. The missions for institutional and external environments are to build an integrated environment for data use, exchange and application. To achieve this goal, fostering communities of specific interests and environments that avail data exchange and knowledge conveyance is essential to external environment planning. For example, discussions within a subjectoriented community that focuses on education, research, ecology, or tourism may encourage information production and consumption. Generally, those communities often assume roles of information 
Sketching environmental information policy and management framework

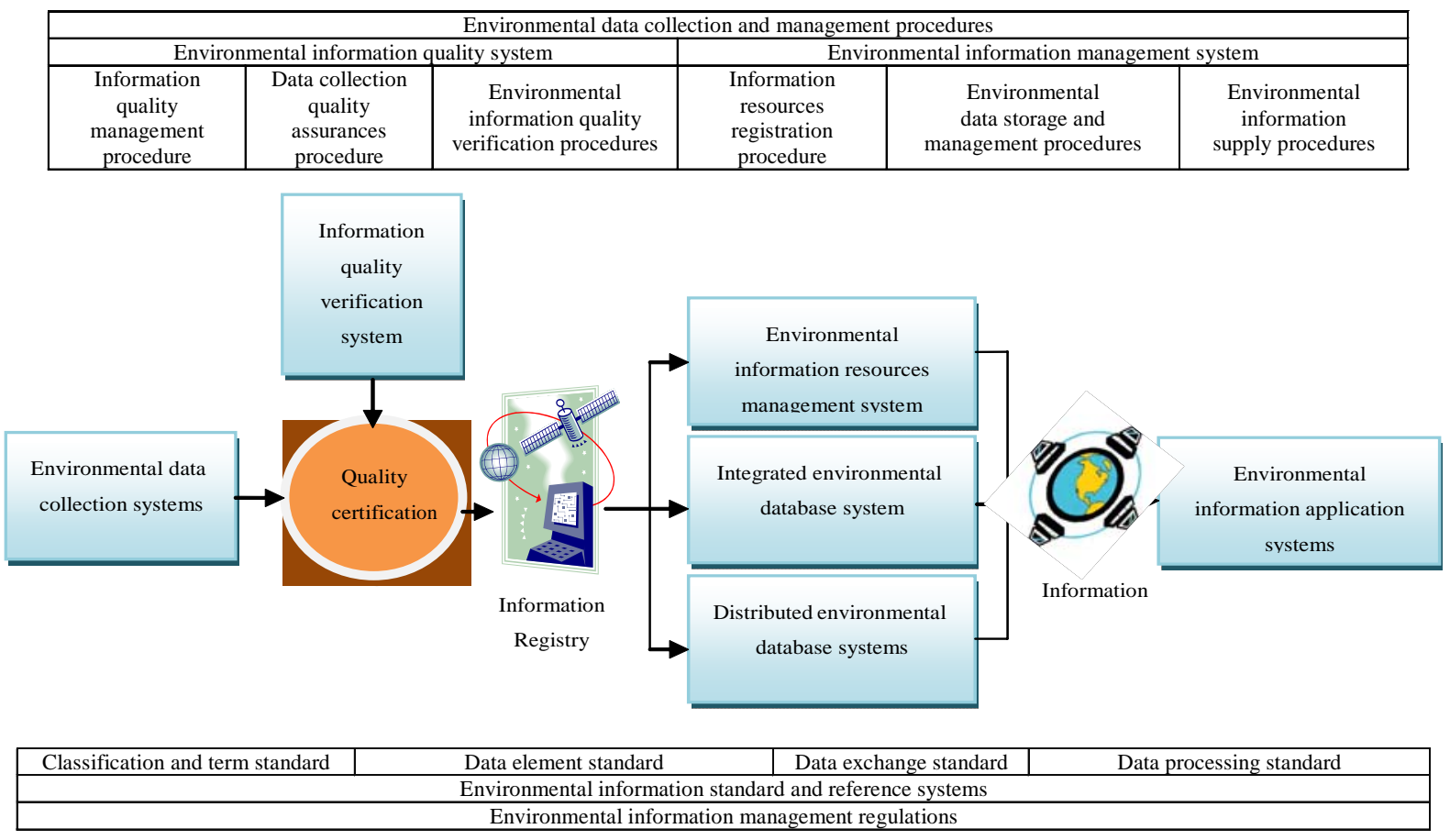

Fig. 5: The conceptual diagram of the distributed environmental databases system

producer and an active community may stimulate information demand. For the communities to acquire germane and accurate environmental information, the producers or suppliers may require to establish corresponding organizational systems such as information management, supply, publish and other knowledge-base and decision-making systems (Tuzkaya and Gulsun, 2008).

In 2002, TWEPA has proposed a long-term plan for establishing a public accessible interface implemented on internet for environmental information publishing and exchange. The information produced by each local county in Taiwan was characteristically and stepwise presented. The following strategy for building the external environment will help the communities of specific interests and environments to stimulate the demand and application of environmental information.

\section{Planning of evolution environment}

The system design is to create an impartial environment to enhance the positive feedbacks between and among entities within environmental information system. Achievement of this ambition requires the consideration of interplay among subjects including employee, organization, citizen and environment. With respect to these subjects, the concern centers in the efficiency of information through the establishment of feedback mechanisms, uniform data entry/storage/provision methods and standards among suppliers and consumers. For example, establishing a genial institutional culture to facilitate the interactions between each producer and consumer of different levels can reduce the latency period of evolution urged by key players that exist in some entities.

This condition will prompt the information distribution as needed and avoid the slackness or asymmetry in information. Because information asymmetry causes unfair competition, it is imperative that system design must include a procedure to periodical assess the performance of each participant. Information quality system often accredits as "natural selection mechanism" in information ecosystem. It can also be viewed as a node, which controls data quantity, quality and 


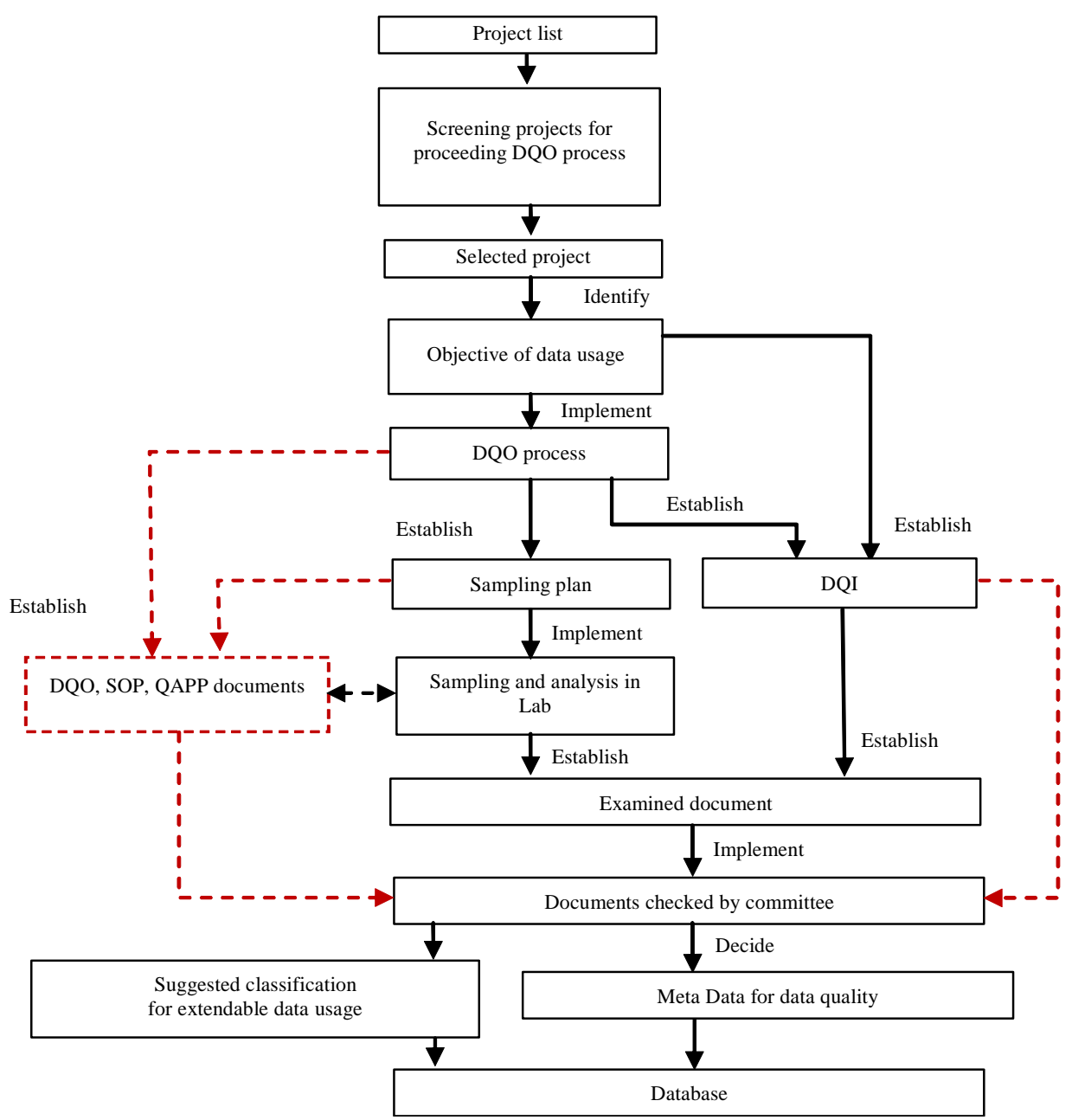

Fig. 6: The systematic flowchart for data quality control

direction, within the network (Fig. 3) information ecosystem esteems distribution and evolution of information and considers competition vital influence on survival of suppliers, consumers and environment. In other words, competition compels suppliers and consumers of poor performance to evolve or otherwise to become extinct. Therefore, an evolution mechanism is essential for governing interactions among information production, consumption and environment. Natural selection intervenes wherever information environment changes; suppliers and consumers that response with necessary institutional reorganizations (such as human resource restructure, altering information strategies, modifying information flows and system architectures and substantiating contents associate with policies and cultures) are most likely to survive in the new environment. Institutional efficiency assessment, external technology changes and competition are primal momentums propelling evolution.

Therefore, creating an evolution environment for environmental information system is critical for managing environmental information. As a support for environmental information ecosystem, evolution environment should be composed of "information policy and efficiency management subsystem" "production quality and management subsystem," "data validation subsystem" "database management subsystem" and "quality identification system" for the purpose of regulating and managing the practice of data production and usage. 
Information policy and efficiency management system

Since environmental information quality system assumes functions of evolution specified in information ecosystem, it is vital to stipulate the quality control guidelines for project/organization campaigning and to establish an efficiency assessment system for auditing personnel, culture, data flows, hierarchies and strategies in the institution and in the intermit, for evaluating human resources, hardware and software configurations.

Once the drawbacks was identified, consequential strategies, such as training programs administration and hardware/software upgrade, for removing impediments toward favorable evolution succession in information environment can be established step by step. To achieve the afore-mentioned, there are some critical actions like 1) establishing an exclusive board of committees; 2) defining guidelines required for organization management and data transportation; 3) establishing an efficiency assessment system, where all individuals should comply with policy analysis, organization management and efficiency assessment of "the information policy and efficiency management system” to be built (Yu et al., 2004).

Data/information production and quality control system

Production and quality control system is to control data production procedures by stipulating necessary specifications and methods pertaining to management and by requesting liable institutes to perform quality control documentation based on the objectives of data production. From the viewpoint of life cycle, data production can be classified into four phases: plan, design, implementation and assessment. These four phases may employ different QA/QC practice, such as data quality objective (DQO), data quality assurance project plan (QAPP), standard operation procedures (SOP) and technical audit (TA). All of these steps in life cycle determine the overall data quality. The mission of quality control system is to ensure the quality requirements for each data objective and to minimize errors occurred in each phase of data production.

From the viewpoint of data production and information application, the quality management should be planned based on the final objective of information production. The relationships between data/information and QA/QC program are shown in Fig. 6. Fig. 6 implies that, in order to deal with the spatial and temporal characteristics in environment and to obtain representative information for decision making, a detailed sampling scheme is needed while extracting information from raw data. When executing a data production plan, DQO process plays a key role in ensuring the achievement of data quality objectives. The purpose of data/information production and quality control system is to ensure the satisfaction of data quality with respect to their designated usage. The extended use of existing information for other management activities is difficult to be regulated. To solve these problems, classification of data/information usage and responsive suggestion on data quality need to be established. Six classes of different data usage were used. This kind of classification also provides the notation of data quality for other intended uses of environmental data. This approach can help to assess the risks or uncertainties associated with information usage and quality control.

\section{Environmental information validation system}

Before storing and applying, data accuracy must be tested against information validation system, which provides procedures or methods for evaluating occasions or subjects of data applications. Then marks or highlights inept and incomplete data; in addition, it should be able to clarify aptness and requirements for utilizing specific environmental data, models and applications for the sake of facilitating data categorization and effortless data flows. The quality validation for environmental data is composed of two categories: procedure validation and reliability validation. Fig. 7 outlines the flowchart of procedure validation that emphasizes the importance of quality control in production. The reliability validation was used to assess applicability of these data in practice. Fig. 8 presents the concept and the approach for implementing the reliability validation. In fact, it is laborious for data managers to do it at present unless the technique for intelligent data mining can be established.

\section{Databases management system}

Data validated by accuracy and aptness analysis can then be stored in database, model-base and 
Int. J. Environ. Sci. Tech., 7 (2), 313-326, Spring 2010

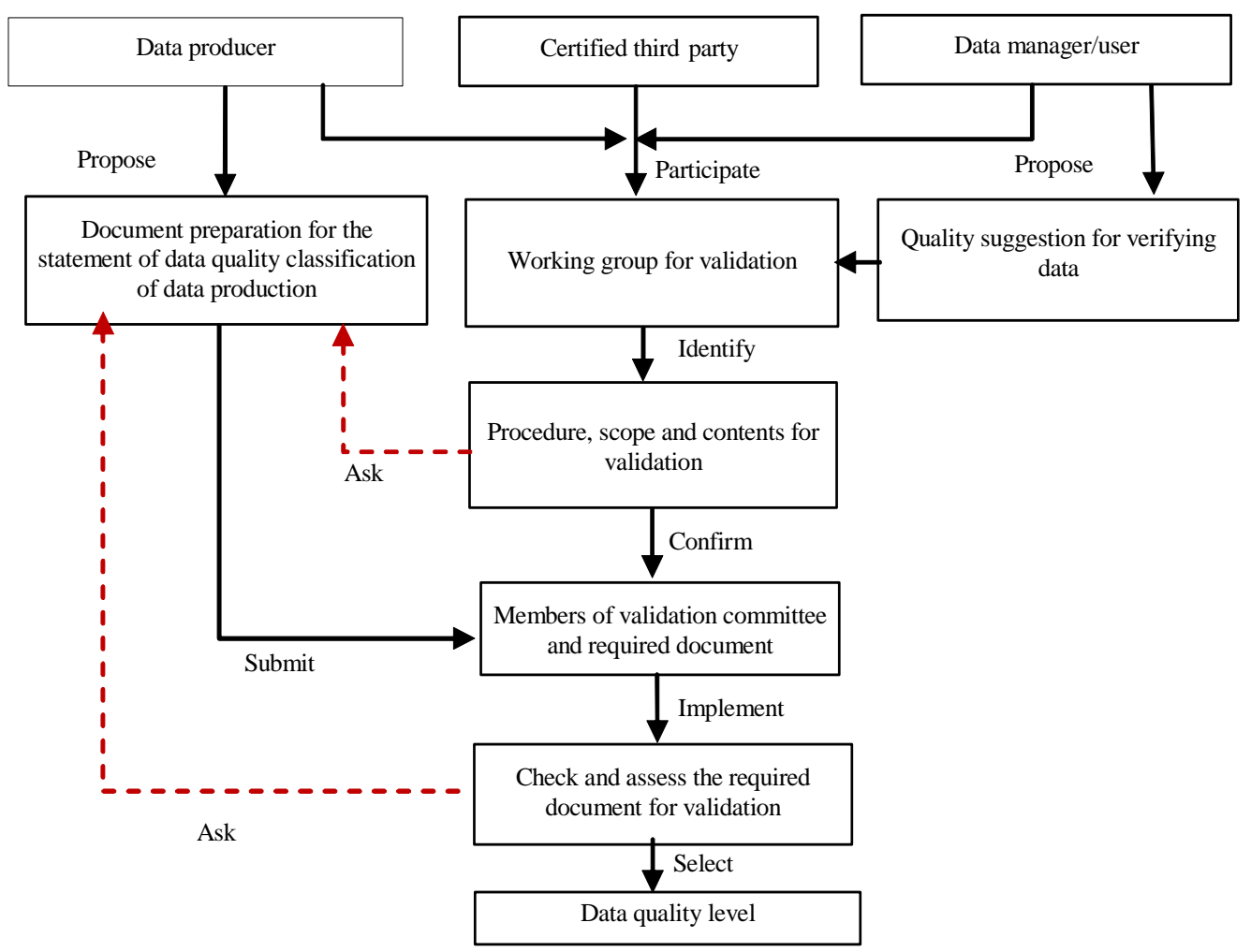

Fig. 7: The flowchart of procedure validation for environmental data quality

knowledge-base. To minimize undesired errors occurred during data storing and ensure data accuracy and suitability, it is essential to fortify/customize database management system and to revise consecutively procedures/methods of data handling/ knowledge updating.

\section{Information certificate system}

Fostering communities of specific interests and providing positive environments for data exchange and knowledge conveyance are essential to external environment planning. For example, discussions within a subject-oriented community that focuses on education, research, ecology, or tourism may encourage information production and consumption. Generally, those communities often play roles of information suppliers; but an active community can sometimes stimulate information demand. In order to supply these communities with germane and accurate environmental information, the producers or suppliers may require to establish corresponding organizational systems such as information management, supply, publish and other knowledge-base and decision-making systems. Since development of future environmental data/ knowledge production probably inclines to produce data for non-academic use, planning policy promoting quality controls over these kinds of data production should be more viable than that for academic data production because of its smaller systematic scale. Data/information production could be undertaken by professional data production of larger-scale (such as those from government and academic institutes), data produced by interested communities with a smaller-medium scale (such as those from organizations concerning tourism/ ecology/environmental protection,) and individual civilians.

Quality control administration based on different levels of data sources is more favorable. For suppliers with larger scale who produces relatively more structural data, controlling over these 
Sketching environmental information policy and management framework

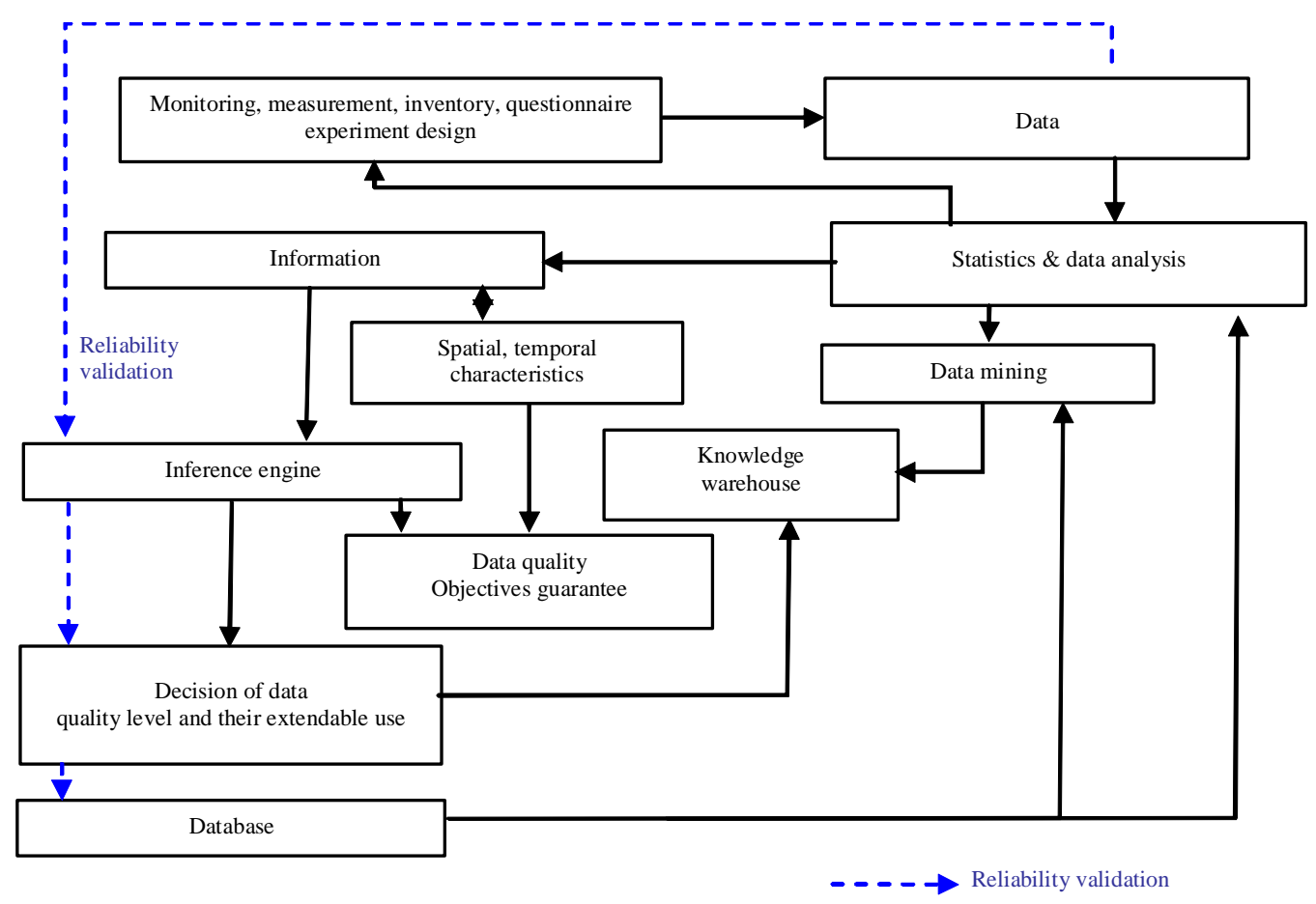

Fig. 8: The flowchart of reliability validation for environmental data quality

production practices should be more efficient because they maybe more experienced in data production. In addition, system of identifying and categorizing data suppliers should be established to reinforce diversity of suppliers and the corresponding data quality. Because diversity of suppliers often tends to foster diversity of consumers, a well-defined mechanism for information quality classification and identification evidently will lead to great variety of roles in handling environmental information. For example, projects funding selective communities concerning different/ specific environment subjects and appropriate rewards may be good options to encourage diversity.

\section{RESULTS AND DISCUSSION}

Objective of environmental data production is to provide relevant data for knowledge-based understanding of environment problems and making decision. To plan an environmental information system regarding merely to suppliers often leads to excessive or inadequate data production of some categories and inappropriate data distribution and applications. Deficit of standardized procedures, lack of evolving mechanism and secluded/disproportional information market often lead to failure of accumulating data into knowledge-base; a capable and continuously evolving information ecosystem is considered to facilitate data communication and knowledge accumulation. According to the concept of information ecology, life cycles of data/information are determined by data consumers or suppliers' ability of data administration/management, which intertwines with their organizational/external environments. On the basis of information ecology, motivation to struggle to survive within a competitive environment will force environmental information system of an organization to evolve automatically; and in return, the efficiency of data usage is promoted.

It is possible that data integrity of an EIS could be compromised by unexpected shutdowns of EIS resulted from discontinued data input. Thus, improving the efficiency/reliability of information exchange and usage in information system is essential 
for establishing a suitable environment for data distribution. Information ecology emphasizes the data use, efficiency, benefit, balance between demand and supply. Thus, the system will promote the information application into many areas in addition to accounting the status of environment.

Facing the rapid change of information environment, this study proposed a systematic framework and a conceptual model for building a more complete information environment to support environmental data/information management. Following the template established in this paper, a more efficient and trustable environmental information flow can be achieved.

\section{ACKNOWLEDGEMENTS}

The authors would like to thank the EPA of the Republic of China for financially supporting this research under Contract No. EPA-91-1601-02-11 and EPA-92-L105-A130

\section{REFERENCES}

Abkowitz, M. D.; Cohen, M. A.; Buck, S. S.; Case, D. W.; Drake, P. A., (1999). Environmental information disclosure and stakeholder involvement: Searching for common ground. Corp. Environ. Strategy, 6 (4), 415-424 (10 pages).

Chen, C. C., (2009). Environmental impact assessment framework by integrating scientific analysis and subjective perception. Int. J. Environ. Sci. Tech., 6 (4), 605-618 (14 pages).

Cranefield, S.; Purvis, M., (2001). Integrating environmental information: incorporating metadata in a distributed information system's architecture. Adv. Environ. Res., 5 (4), 319-325 (7 pages).

Davenport, T. H.; Prusak, L., (1997). Information ecology: Mastering the information and knowledge environment. Oxford University Press, New York.

de Brisis, K., (1995). Government policy for information resources management and its implication for provision of information services to the public and to the experts. Computers, Environ. Urban Syst., 19 (3), 141-149 (9 pages).

Garvey, P., (1998). Environmental protection agency provides internet users with environmental information through web-enabled data warehouse. Appl. Occup. Environ. Hygiene, 13 (4), 216-220 (5 pages).

Gil-Garcia, J. R.; Pardo, T. A., (2005). E-government success factors: mapping practical tools to theoretical foundations. Gov. Inform. Q., 22 (2), 187-216 (30 pages).

Graham, A. W., (1985). The two ecologies: Population and community perspectives on organizational evolution. Admin. Sci. Quart., 30 (2), 224-241 (18 pages).

Haklay, M. E., (2003). Public access to environmental information: Past, present and future. Computers, Environ. Urban Syst., 27 (2), 163-180 (18 pages).
Huang, P. S.; Shih, L. H., (2008). Effective environmental management through environmental knowledge management. Int. J. Environ. Sci. Tech., 6 (1), 35-50 (16 pages).

Huh, Y. U., (1990). Data quality. Infor. Software Tech., 32(8), 559-565 (7 pages).

Karmarkar, U. S.; Apte, U. M., (2007). Operations management in the information economy: Information products, processes, and chains. J. Oper. Manage., 25 (2), 438-453 (6 pages).

Kuchka, H. E., (2001). Method for theory: A prelude to human ecosystems. J. Ecol. Anthropol., 5 (Special), 3-78 (76 pages).

Leire, C.; Thidell, A., (2005). Product-related environmental information to guide consumer purchases - a review and analysis of research on perceptions, understanding and use among Nordic consumers. J. Clean. Produc., 13 (10-11), 1061-1070 (10 pages).

Moreton, R.; Simon, E.; Sloane, A., (1995). Implementing information management and technology standards: A framework. Tech. Manage., 2 (6), 275-288 (14 pages).

Mutula, S.; Wamukoya, J. M., (2009). Public sector information management in east and southern Africa: Implications for FOI, democracy and integrity in government. Int. J. Inform. Manage., 29 (5), 333-341 (9 pages).

Nalle, D. J.; Montgomery, C. A.; Claire, A. J.; Arthur, L.; Polasky, S. S., (2004). Modeling joint production of wildlife and timber. J. Environ. Econ. Manage., 48 (3), 997-1017 (21 pages).

Prokopiadou, G.; Papatheodorou, C.; Moschopoulos, D., (2004). Integrating knowledge management tools for government information. Govern. Inform. Q., 21 (2), 170198 (19 pages).

Purvis, M.; Cranefield, S.; Ward, R.; Nowostawski, M.; Carter, D.; Bush, G., (2003). A multi-agent system for the integration of distributed environmental information. Environ. Modell. Softw., 18 (6), 565-572 (8 pages).

Reddick, C. G., (2005). Citizen interaction with e-government: from the streets to servers? Govern. Inform. Q., 22 (1), 38-57 (20 pages).

Scholl, H. J., (2006). Electronic government: Information management capacity, organizational capabilities and the sourcing mix. Govern. Inform. Q., 23 (1), 73-96 (24 pages).

Seifert, J. W., (2004). Data mining and the search for security: Challenges for connecting the dots and databases. Govern. Inform. Q., 21(4), 461-480 (20 pages).

Sofia Pereira, C.; Soares, A. L., (2007). Improving the quality of collaboration requirements for information management through social networks analysis. Int. J. Inform. Manage., 27 (2), 86-103 (18 pages).

Steep, J. R., (1999). Prospectus for information ecology. Georg. J. Ecol. Anthropol., 3, 38-54 (17 pages).

Szaro, R. C.; Berc, J.; Cameron, S.; Cordle, S.; Crosby, M.; Martin, L.; Norton, D.; O’Malley, R.; Ruark, G., (1998). The ecosystem approach: Science and information management issues, gaps and needs. Landscape Urban Plan., 40 (1-3), 89-101 (13 pages).

Tuzkaya, G.; Gülsün, B., (2008). Evaluating centralized return centers in a reverse logistics network: An integrated fuzzy 
multi-criteria decision approach. Int. J. Environ. Sci. Tech., 5 (3), 339-352 (14 pages).

TWEPA, (2002). A planning and setting of metadata system, managed and public method, local environmental database in environmental database system and advertisement in taiwan (EPA-91-L105-02-214), Taiwan. (in Chinese).

USEPA, (2002a). Overview of the EPA Quality System, Office of Environmental Information, (EPA/240/R-02/003), United States Environmental Protection Agency. Washington, DC 20460.

USEPA, (2002b). Policy and Program Requirements for the Mandatory Agency-wide Quality, Office of Environmental Information (EPA Order 5360.1 A2), United States Environmental Protection Agency. Washington, DC 20460.
Wang, R. Y.; Reddy, M. P.; Kon, H. B., (1995). Toward quality data: an attribute-based approach. Decis. Support Syst., 13, 349-372 (24 pages).

Wang, R. Y.; Strong, D. M., (1996). Beyond accuracy: what data quality means to data consumers? J. Manage. Syst., 12, 5-34 (30 pages).

Ward, M.; Mitchell, S., (2004). A comparison of the strategic priorities of public and private sector information resource management executives. Govern. Inform. Q., 21 (3), 284304 (21 page).

Yu, R. F.; Chen, H. W.; Cheng, W. P.; Chi, F. H.; Liaw, S. L.; Yu, Y. H., (2004). Development of a data quality management system for ROC EPA. J. Chinese Inst. Environ. Eng., 14(3), 141-149 (9 pages).

\section{AUTHOR (S) BIOSKETCHES}

Chen, H. W., Ph.D., Department of Environmental Engineering and Management, Chaoyang University of Technology (CYUT), 168 Jifong E. Rd., Wufong Township Taichung County, 41349, Taiwan (R.O.C.). Email: hwchen@cyut.eud.tw

Yu, R. F., Ph.D., Department of Safety, Health and Environmental Engineering, National United University, No. 1 Lien-kung, Kung-ching Li, Miaoli, Taiwan 360, Republic of China. Email: rfyu@nuu.edu.tw

Liaw, S. L., Ph.D., Graduate Institute of Environmental Engineering, National Central University, Taiwan, No.300, Jhongda Rd., Jhongli City, Taoyuan County 32001, Taiwan (R.O.C.). Email: slliaw@ncuen.ncu.edu.tw

Huang, W. C., Ph.D. Candidate, Graduate Institute of Environmental Engineering, National Central University, Taiwan, No.300, Jhongda Rd., Jhongli City, Taoyuan County 32001, Taiwan (R.O.C.). Email: wanchi@esam.ev.ncu.edu.tw

Chen, H. W.; Yu, R. F.; Liaw, S. L.; Huang, W. C., (2010). Information policy and management framework for environmental protection organization with ecosystem conception. Int. J. Environ. Sci. Tech., 7 (2), 313-326. 